\title{
Preparation, Characterization and Electroluminescence Studies of Cadmium Selenide Nanocrystals
}

\author{
Reena Tekchandani, Nitendra Gautam, Meera Ramrakhiani*
}

Department of Post Graduate Studies and Research in Physics and Electronics Rani Durgavati University, Jabalpur, INDIA

\begin{abstract}
Nanoparticles of CdSe were prepared using chemical method by reacting cadmium acetate and sodium selenosulfite in presence of capping agent mercaptoacetic acid (MAA). Two-propanol was added to this mixture, drop wise, till it becomes turbid. The precipitate was separated through centrifugation to get dry powder.

TEM shows that the most particles are spherical with particle size of the order of few nanometres. The XRD pattern indicates the hexagonal phase with wurtzite structure. The broadening of peaks tends to increase with increasing capping agent concentration showing decrease in particle size in range of $3 \mathrm{~nm}$ to $4 \mathrm{~nm}$. Absorption spectra of CdSe nanoparticles indicated that the position of absorption peak shift to smaller wavelength as the capping agent concentration increases. Considering this as band-to-band absorption, their effective band gap was calculated and particle size was estimated using effective mass approximation, which is nearly same as obtained by XRD.

The electroluminescence (EL) cells were prepared by placing CdSe nanoparticles between $\mathrm{SnO}_{2}$ coated conducting glass plate and aluminum foil. Alternating voltage of various frequencies was applied and EL brightness at different voltages was measured and corresponding current was also recorded. It is seen that emission starts at a threshold voltage and then increases rapidly with increasing voltage. The lower threshold and higher brightness have been observed for smaller nanoparticles. Linear relation between voltage and current indicates ohmic nature. Voltage vs brightness curve shows that EL is produced by acceleration-collision mechanism.
\end{abstract}

Keywords: CdSe Nanocrystals, Absorption Spectra and Electroluminescence.

\section{INTRODUCTION}

The novel optical and electrical properties of nanomaterials can be controlled as per their particle size and therefore attracted much interest for their fundamental and applied aspects [1]. Because of the novel physical properties and potential applications semiconductor nanoparticles have been studied extensively [2-4]. As a new type of optical material, semiconductor nanoparticles present very unique properties. Due to quantum size effect, the absorption and fluorescence spectra of the quantum size semiconductor particles can be greatly improved by modifying the particle surface [5]. These advantages make nanostructured material an ideal candidate for the use in electroluminescence applications [6]. Cadmium selenide (CdSe) is a well known luminescent material and is being used in various devices. It is used as phosphor and also in thin film electroluminescent devices. The effect of reducing the size of CdSe crystals is expected to improve the performance of these devices and also their characteristics can be tailored [7]. CdSe quantum dots exhibit size dependent tunable photoluminescence with broad excitation spectra and narrow emission band width that span the visible spectrum. Therefore they are potentially useful in

*Address correspondence to this author at the Department of Post Graduate Studies and Research in Physics and Electronics Rani Durgavati University, Jabalpur INDIA; TeL: 09977545922; E-mail: mramrakhiani@hotmail.com a wide range of applications [8]. Present studies have been undertaken to synthesize CdSe nanocrystals of various sizes and investigated their electroluminescence.

\section{EXPERIMENTAL METHODS}

\subsection{Synthesis of Samples}

Synthesis of CdSe nanocrystal is based on reaction of cadmium acetate and sodium selenosulfite in presence of mercapto acetic acid as capping agent [9]. A solution of 2 $\mathrm{mMol}$ concentration of cadmium acetate and mercapto acetic acid were mixed in $400 \mathrm{ml}$ water and $\mathrm{NaOH}$ aqueous solution was added to set the $\mathrm{pH}$ at 8.5 . Resulting solution was deaeraced with $\mathrm{N}_{2}$ bubbling for 30 minute. The solution of $0.02 \mathrm{M}$ sodium selenosulfite $\mathrm{Na}_{2} \mathrm{SeSO}_{3}$ was prepared by refluxing an aqueous solution containing $0.2 \mathrm{M}$ sodium sulfite and $0.02 \mathrm{M}$ selenium powder for 30 minutes under vigorous stirring. $10 \mathrm{ml}$ of freshly prepared oxygen free $0.02 \mathrm{M}$ $\mathrm{Na}_{2} \mathrm{SeSO}_{3}$ was added to mixture of mercapto acetic acid (MAA) and cadmium acetate solution. The size selective precipitation method was used to precipitate the CdSe from the crude solution. They were concentrated down approximately $1 / 5$ of original solution using a rotatory evaporator. 2propanol was added drop wise in the resulting solution with the help of burette. The solution was again left for 24 hours to let the precipitate settle down in the bottom of the flask. The solution was centrifuged for 30 minutes at $3500 \mathrm{rmp}$. 


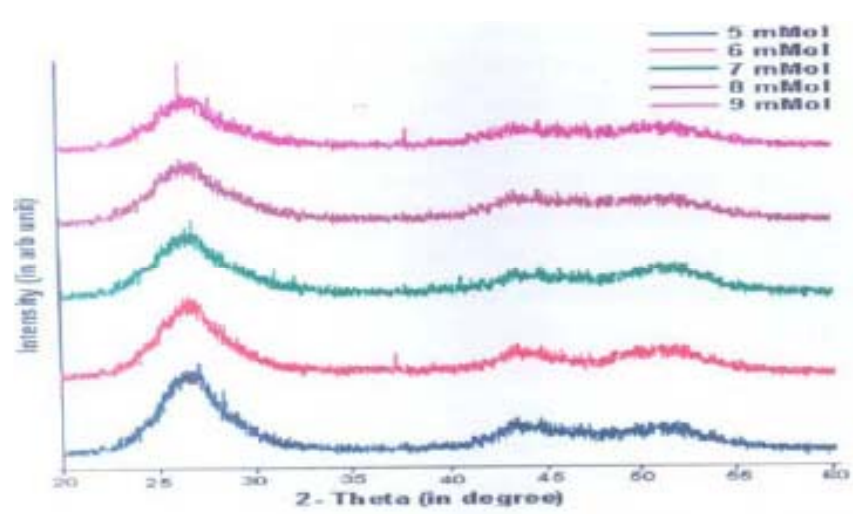

Fig. (1). XRD patterns of CdSe nanocrystals.

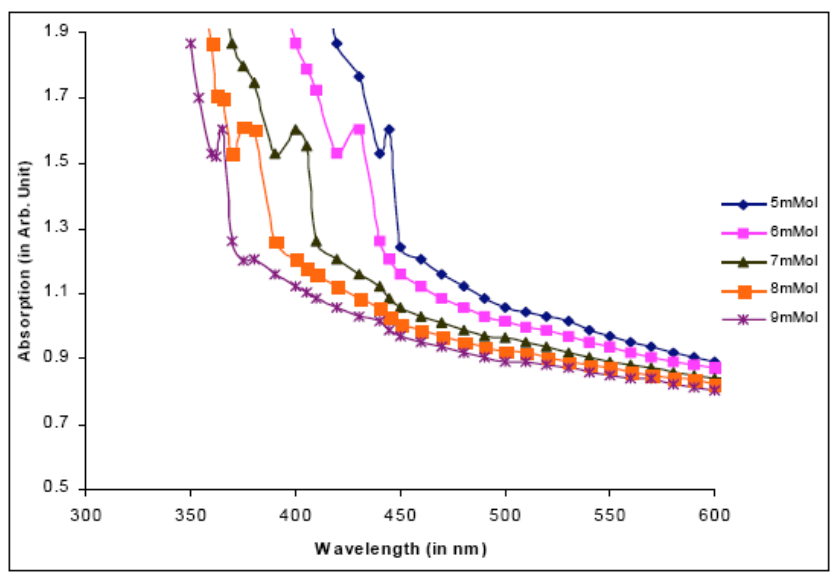

Fig. (2). Absorption spectra of CdSe nanopartticles.

Finally the precipitate was spread over a glass substrate and air-dried at room temperature. The capping agent concentration controls the size of nanocrystals. For present investigation, five different samples were prepared by using different concentration of capping agent varying from 5 through 6,7 , 8 and $9 \mathrm{mMol}$.

\subsection{Characterization}

The samples were characterized for morphology by TEM using Techani $20 \mathrm{G}$ electron microscope at IUC Indore. X-ray diffraction studies have been undertaken for all the samples in order to determine their crystal structure and crystalline size. X-ray diffraction patterns have been obtained by $\mathrm{Ri}$ gaku Rotating Anode (H-3R) diffractometer with irradiation from $\mathrm{K}_{\alpha}$ line of copper $(\lambda=1.5418 \AA)$. Optical absorption studies carried out on a Perkin Elemer lambda -12 spectrometer.

\subsection{EL Measurement}

The EL cells were prepared having a triple layer structure namely a nanocrystals emission layer, sandwiched between two electrodes. The transparent electrode has been prepared by depositing of thin film of $\mathrm{SnO}_{2}$ by chemical vapor deposition on clean glass substrate. This $\mathrm{SnO}_{2}$ coated glass behaves as an electrode. Nanoparticles of $\mathrm{CdSe}$ were mixed in DMSO (di methyle sulfo oxide) and spread over small portion of $\mathrm{SnO}_{2}$ coated conducting glass and was allowed to dry. Then a mica sheet having a window of $2 \times 2 \mathrm{~mm}$ was placed on the conducting glass plate so that window is on the film of CdSe sample. An Aluminum foil strip was placed tightly over this window so as to have good contact with CdSe nanoparticle film and there is no air gap. The aluminum strip acts as second electrode. The layer of nanoparticle should be uniform and thin. Alternative voltage of various frequencies was applied and EL brightness (B) at different voltages (V) was measured with the help of photomultiplier tube and corresponding current was also recorded.

\section{RESULTS AND DISCUSSION}

TEM images of CdSe nanoparticles show that these are nearly spherical in shape. It is seen that, as expected, particle size decreases with increase in capping agent concentration.

Fig. (1) shows the diffraction patterns of CdSe nanoparticles prepared with different concentration of mercaptoacetic acid used as capping agent. Three peaks are obtained in all the samples.

The particle size has been determined using DebyeScherrer formula [10] from the first peak. It is observed that the diffraction patterns of nanoparticles are broadened as the capping agent concentration is increased. The crystalline size computed from XRD peaks come out to be in the range of 3 $\mathrm{nm}$ to $4 \mathrm{~nm}$. The particle size obtained for various concentration of capping agent is shown in Table-I which shows that particle size of $\mathrm{CdSe}$ decreases with increasing capping agent.

Fig. (2) shows the UV/VIS optical absorption spectra for $\mathrm{CdSe}$ nanocrystals in the range $600 \mathrm{~nm}$ to $300 \mathrm{~nm}$. It can be seen from the spectra that there is slight increase

in absorbance starting from 600 to $450 \mathrm{~nm}$. Further decreasing the wavelength from 450 to $350 \mathrm{~nm}$, there is fast increase in absorbance, a peak and then again fast increase in absorbance at lower wavelengths. For CdSe-I sample, increase in the absorption occurred at about $450 \mathrm{~nm}$ and peak of absorption is obtained at about $445 \mathrm{~nm}$. Similarly the absorption peaks for other samples are obtained at 430, 400, $380,365 \mathrm{~nm}$ for $\mathrm{CdSe}-\mathrm{II}$ to $\mathrm{CdSe}-\mathrm{V}$ samples respectively. Considering this as band to band transition their effective band gaps were calculated and given in Table $\mathbf{1}$.

Fig. (2). clearly shows that the absorption spectra of $\mathrm{CdSe}$ nanoparticles shift towards shorter wavelengths by increasing MAA concentration or decreasing size. Effective mass approximation (EMA) model is used to estimate the particle size from the increase in effective band gap of nanocrystals, which are nearly same as obtained by XRD.

Optical excitation of electrons across the band gap is strongly allowed transition, producing an abrupt increase in absorptivity at the wave length corresponding to the gap energy. This feature in the absorption spectrum is known as the optical absorption edge. In case of nanocrystals, the electronic energy levels are discretized and effective band gap energy increases [11]. As the particle size is reduced the absorption edge shifts towards blue side and absorption peak may be obtained due to discrete energy levels as in present case. A graph is plotted between MAA concentration and the band gap and particle size corresponding to the absorption peak (Fig. 3). 
Table I. Particle size of CdSe Nanoparticles by XRD, TEM and EMA

\begin{tabular}{|c|c|c|c|c|c|c|}
\hline S.No. & Sample & $\begin{array}{c}\text { Concentration of Capping } \\
\text { Agent in (mMol) }\end{array}$ & $\begin{array}{c}\text { Size by XRD } \\
\text { (in nm) }\end{array}$ & $\begin{array}{c}\text { Size by TEM } \\
\text { (in nm) }\end{array}$ & $\begin{array}{c}\text { Band gap E } \\
\text { (eV) }\end{array}$ & $\begin{array}{c}\text { Size by EMA } \\
\text { (in nm) }\end{array}$ \\
\hline \hline 1 & CdSe -I & 5 & 3.85 & $5.2 \mathrm{~nm}$ & 2.78 & 3.8 \\
\hline 2 & CdSe -II & 6 & 3.56 & $4.9 \mathrm{~nm}$ & 2.88 & 3.6 \\
\hline 3 & CdSe -III & 7 & 3.28 & $4.1 \mathrm{~nm}$ & 3.1 & 3.3 \\
\hline 4 & CdSe -IV & 8 & 3.15 & - & 3.26 & 3.1 \\
\hline 5 & CdSe -V & 9 & 3.09 & - & 3.39 & 3.01 \\
\hline
\end{tabular}

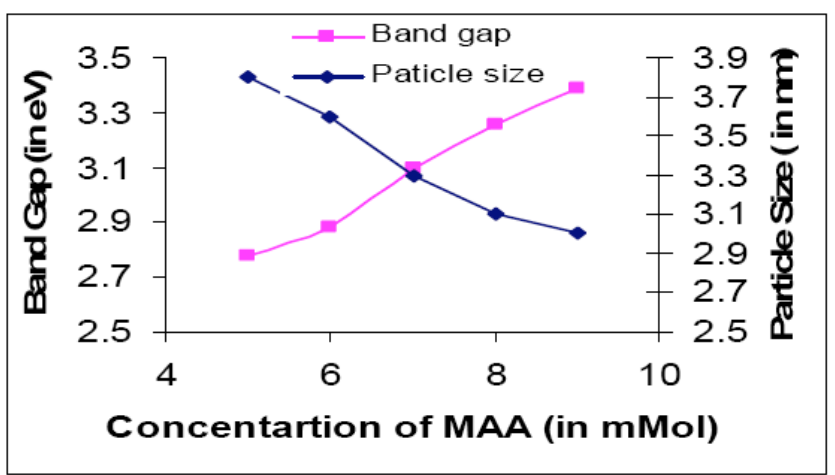

Fig. (3). Variation of band gap and particle size with MAA concentration.

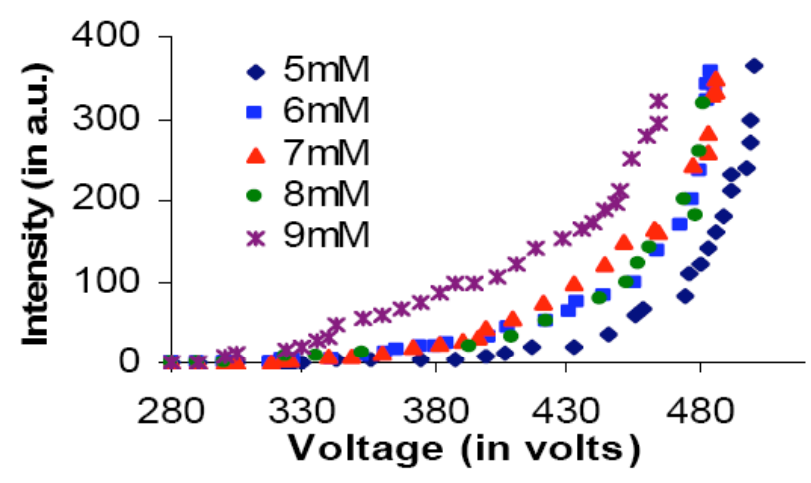

Fig. (4). Voltage-Brightness Characteristics of CdSe nanoparticles.

As the capping agent concentration increases, band gap is found to increase and particle size is reduced but the effect is not linear. It is observed that no optical absorption occurs at surface states and therefore these do not affect the absorption spectra. Only widening of band gap is indicated.

Similar results are reported by Ashtaputure et al., [12] who have prepared $\mathrm{CdSe}$ nanoparticles using chemical route method and 2-merceptoethanol as a capping agent. The samples show excitonic peaks at 360 and $385 \mathrm{~nm}$ corresponding to particle sizes of 2.8 and $3.7 \mathrm{~nm}$, showing a clear blue shift. Sondi et al., [13] prepared Amdex CdSe nanoparticles with aqueous solutions of $\mathrm{Na}_{2} \mathrm{Se}$ and $\mathrm{CdCl}_{2}$, in the presence of different concentrations of Amex. They found that increasing the concentration of Amex resulted in a shift of the onset of the absorption towards shorter wave lengths, indicating a decrease in the size of CdSe nanoparticle. The peaks shifted from 450 to $420 \mathrm{~nm}$.

The EL studies of various CdSe nanocrystalline samples have shown that light emission occurs at certain threshold

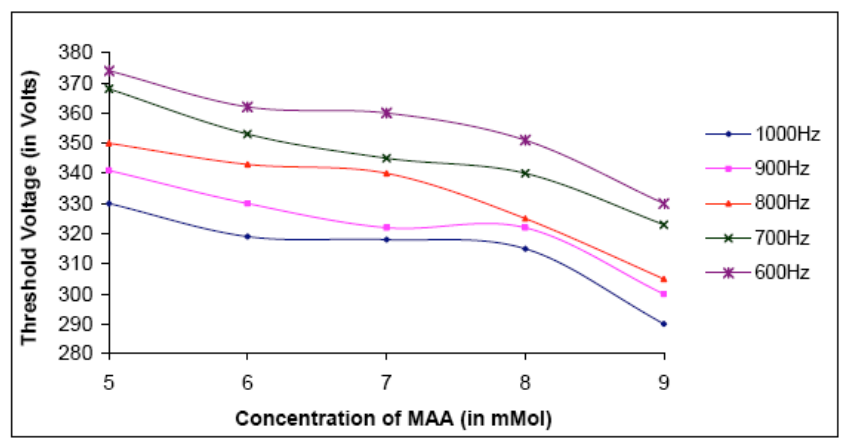

Fig. (5). Variation of Threshold Voltage with MAA concentrations.

voltage and then increases rapidly with increase in voltage. The voltage brightness characteristics at different concentration of capping agent (MAA) and fixed $\mathrm{pH}$ value (8.5), for frequency $1 \mathrm{KHz}$ are shown in Fig. (4).

The lower threshold voltage and higher brightness have been observed for high MAA concentration. Fig. (5) shows the variation in threshold voltage at different frequencies by changing the particle size of CdSe nanocrystals. It is clear from the figure that, as MAA concentration increases or $\mathrm{CdSe}$ particle size decreases, threshold voltage also decreases. By measuring EL brightness (B) at particular voltage $\mathrm{V}$ and frequency, it is seen that higher brightness is obtained for smaller particles.

EL of CdSe nanoparticles of different concentration of MAA ( $5 \mathrm{mMol}$ to $9 \mathrm{mMol}$ ) were observed at different frequencies stating from $600 \mathrm{~Hz}$ to $1000 \mathrm{~Hz}$ and found that on increasing the frequency of input ac signal EL brightness increases continuously. The relation between current and voltage is found to be linear. This indicates ohmic contact between the sample and the electrodes. From the slope of the $\mathrm{I}-\mathrm{V}$ characteristics it is found that the impedance decreases with increasing frequency.

Nanocrystal properties lie between those of molecules and bulk materials. Due to three-dimensional carrier confinement and a large surface to volume ratio, energy relaxation and recombination dynamics in nanocrystals are significantly different from those in bulk materials. As molecules, nanocrystals can interact with electric field via their electronic transition dipoles, but because of their solid state nature, they exhibit unusual photo physics relative to molecules. In the electroluminescence (EL) process electrons from the valence band are excited to the conduction band on applying the electric field. These excited electrons can be deexcited through non-radiative recombination to some surface or defect state [14]. It is natural to consider surface located 
native defects such as cadmium vacancies while discussing the nature of the defect states contributing to the band-edge electroluminescence. The deep-trap emission in CdSe nanocrystals can be assigned to a surface cadmium vacancy with a level located below the conduction band. A Cd vacancy can create two acceptor states in the energy gap: one (single ionized) above the valence band and the other (doubly ionized) below the conduction band [15]. Bulk CdSe is an n-type material with a conductivity dominated by foreign donor atoms. However, different situations in nanocrystals with large number of surface located native defect can lead to the existence of uncompensated acceptors along with compensated ones.

The same pattern of V-I graph and EL versus voltage graph has been found in our case except that for low particle size of CdSe, low voltage is required to produce current through the samples and light emission from the films. It is observed that EL starts at threshold voltage and then increases first slowly and then rapidly with the increase in voltage. The lower threshold and higher brightness have been observed for smaller CdSe particles. As voltage is increased, more electrons and holes are injected into the emission layer and their subsequent recombination increases the EL brightness. For higher MAA concentration, smaller CdSe nanoparticles are obtained having increased oscillator strength, which improves the electron-hole radiative recombination and enhance the electroluminescence. It is observed that at high frequencies, light emission starts at lower threshold voltages, and electroluminescence brightness increases with increasing frequency. The nature of frequency dependence of EL brightness can be understood on the basis that the emptying and detrapping of EL centers more rapidly with increase in frequency. The other reason may be due to that, by decreasing the particle size, the nanoparticles of $\mathrm{CdSe}$ becomes more pure. This acts as a good charge transfer material. The space charge air gap also reduces, which results in emitting brightness at low voltage for smaller particle size. The current-voltage characteristics of EL cells indicate ohmic nature.

The similar results have been found out by V.Nogriya et al., [16] in which CdS nanocrystals were prepared by single source organometallic precursor. The EL studies showed that the light emission started at some threshold voltage and then increases rapidly with increasing applied voltage. Smaller CdS crystals gave EL of higher brightness starting at low threshold voltage. It was observed that the EL in $\mathrm{CdS}$ nanoparticles arises due to the high electric field, in which band bending caused the release of trapped electrons and their subsequent recombination with holes gave rise to the light emissions. Kushwaha et al., [17] have also reported electroluminescence of CdSe nanoparticles in polyvinyl alcohol. They have found that smaller CdSe particles prepared at higher $\mathrm{pH}$ give EL in low voltage range.

Although a number of empirical and theoretical relation have been proposed, the most suited relation for the voltage dependence of EL brightness may be given by [18]

$\mathrm{B}=\mathrm{B}_{0} \exp (-\mathrm{b} / \sqrt{\mathrm{V}})$ where $\mathrm{B}_{0}$ and $\mathrm{b}$ are constant which depends on temperature and frequency of the applied voltage and material of the EL cell.

For CdSe nanoparticles, the equation (1) successfully explains the voltage-brightness characteristic. The curves between $\log (\mathrm{B})$ and $100 / \sqrt{ } \mathrm{V}$ has been found to be straight line with negative slope. This indicates that EL is produced by acceleration-collision mechanism.

\section{CONCLUSION}

The studies have shown that smaller CdSe nanocrystals can be prepared by increasing capping agent concentration. Blue shift in absorption peak indicates increase in effective band gap. Electroluminescence studies have shown that in case of smaller particles, higher EL intensity is obtained and light emission starts at lower threshold. Thus high efficiency EL devices for display and lighting can be fabricated using smaller CdSe particles prepared by higher MAA concentration. Color of emission may also be changed by changing the particle size.

\section{ACKNOWLEDGEMENT}

The authors gratefully acknowledge the assistance provided by Inter University Consortium (IUC), Indore for characterizing the samples by TEM and XRD.

\section{CONFLICT OF INTEREST}

None.

\section{REFERENCES}

[1] Zhong, Q.Y.; Cun, L.; Ning, Z. Size dependence of the luminescence spectra of nanocrystal alumina. J. Lumin., 2002, 99, 29-34.

[2] Jonathan, D. G.; Michael, A. S.; Michael, J. B. II; Sandra, J. R.; Sharon, M.W. Cadmium selenide nanocrystals as white light phosphors. Proc. SPIE., 2006, 6337, 63370A.

[3] Zheng, Y.; Yang, Z.; Ying. Y.J. Aqueous synthesis of glutathione capped $\mathrm{ZnSe}$ and $\mathrm{Zn}_{1-x} \mathrm{Cd}_{x}$ Se Alloyed Quantum dots. Adv. Mater, 2007, 19, 1475.

[4] Chauksey, P.; Chandra, B.P.; Ramrakhiani M. Electroluminescence of $\mathrm{CdS}$ nanoparticle-polyvinyl carbazole composites. Indian. J. Eng. Mater. Sci. 2009, 16, 157.

[5] Hines, M.A.; Guyot-sionnest, P. Synthesis and characterization of strongly luminescent ZnS-capped CdSE nanocrystals. J. Phys. Chem. 1996, 100, 468- 471.

[6] Gao, M.; Richer, B.; Kirstein, S.; Mohwald, H. Electroluminescence studies on self assembled films of PP and CdSe nanoparticles. J. Phys. Chem. B, 1998, 102, 4096-4103.

[7] Bandaranayake, R.J.; Wen, G.W.; Lin, J.Y; Jain, H.X.; Sorensen, C.M. Structural phase behavior in II-VI semiconductor nanoparticles. Appl. Phys. Lett. 1995, 67(6), 831-833.

[8] Liu, H.W.; Laskar, I.R.; Haung, C.P.; Cheng, J.A.; Cheng, S.S.; Lao, L.Y.; Wang, H.R.; Chen, T.M. Enhanced phosphorescence and electroluminescence in triplet emitter by doping gold into cadmium selenide/zinc sulfide nanoparticles. Thin Solid Films, 2005, 489, 296-302.

[9] Zhang, S.; Yu, J.; Li, X.; Tian, W. Photoluminescence properties of mercaptocarbolic acid stabilized CdSe nanoparticles covered with polyelectroliyte. Nanotechnology, 2004, 15, 1108-1112.

[10] Cullity, B. Elements of X-Ray diffraction, II Ed. Addison-Wesley, Redding. 1978

[11] Sahu, S.N.; Nanda, K.K. Nanostructure semiconductors: physics and applications. PINSA, 2001, 67 A, 103-130.

[12] Ashtaputure, S.S.; Urban, J.; Haram, S.K.; Gosavi, S.W.; Kulkarni, S.K.; Synthesis and analysis of $\mathrm{ZnO}$ and $\mathrm{CdSe}$ nanoparticles. J.Phys. 2005, 65, 615-617. 
[13] Sondi, I.; Siiman, O.; Matijevic, E. Synthesis of CdSe nanoparticles in the presence of aminodextran as stabilizing and capping agent. $J$. Colloid. Interface Sci. 2004, 275, 503-507.

[14] Wilson, J.; Hawkes, J.F.B. Prentice-Hall India Pvt.Ltd. Optoelctronics: An introduction. II Ed, 1999, 122-126.

[15] Lorenz, M.R.; Woodbury, H.H. Double acceptor defect in CdTe. Phys.Rev.Lett. 1963, 10(6), 215-217.

[16] Nogriya,V.; Dongre, J.K.; Ramrakhiani, M.; Chandra, B.P. Electroand photo-luminescence studies of CdS nanocrystals prepared by organometallic precursor. Chalcogenide Letters; 2008, 5(12), 365373.

[17] Kushwaha, K.; Shukla; Priyanka; Durgesh, N.; Singh, P.; Ramrakhiani. Electroluminesce from CdSe/PVA nanocomposites. M.; Nano Vision, 2011, 1(2), 64.

[18] Kalinowski, J. Electroluminescence in organics. J. Phys. D: Appl. Phys. 1999 32, R179-R250.

Received: August 07, 2011

Revised: September 21, 2011

Accepted: October 04, 2011

() Tekchandani et al.; Licensee Bentham Open.

This is an open access article licensed under the terms of the Creative Commons Attribution Non-Commercial License (http://creativecommons.org/licenses/by-nc/3.0/) which permits unrestricted, non-commercial use, distribution and reproduction in any medium, provided the work is properly cited. 